\title{
Catechol Oxidation by Peroxidase-positive Astrocytes in Primary Culture: An Electron Spin Resonance Study
}

\author{
Hyman M. Schipper, ${ }^{1}$ Yashige Kotake, ${ }^{2}$ and Edward G. Janzen ${ }^{2}$ \\ 'Department of Neurology, McGill University, Lady Davis Institute for Medical Research of the Sir Mortimer B. Davis- \\ Jewish General Hospital, Montreal, Quebec, Canada H3T 1 E2 and ${ }^{2}$ Departments of Clinical Studies and Biomedical \\ Sciences, University of Guelph, Guelph, Ontario, Canada N1G 2W1
}

In rodents, chronic estrogenization has been shown to induce degeneration of dendrites and myelin figures in the hypothalamic arcuate nucleus adjacent to peroxidase-positive astrocyte processes. Because in this brain region estradiol is metabolized to 2-hydroxyestradiol (catecholestrogen), we hypothesized that the latter may be oxidized by the astrocytic peroxidase activity to cytotoxic ortho-semiquinones as occurs in peripheral tissues. Cysteamine induces nonenzymatic peroxidase activity in cultured astroglia identical to that observed in vivo. Using electron spin resonance, we demonstrate robust peroxidase-catalyzed oxidation of 2-hydroxyestradiol and dopamine by cysteaminepretreated astrocyte cultures relative to untreated controls. These results implicate the peroxidase-positive astrocytes in the pathogenesis of estradiol-related hypothalamic damage, parkinsonism, and other free-radical-related neurologic disorders.

A distinct subpopulation of granule-laden astrocytes exhibiting an affinity for chrome alum hematoxylin and aldehyde fuchsin (Gomori stains) has been described in the periventricular brain of many vertebrates, including humans. Their cytoplasmic inclusions are rich in sulfhydryl groups, emit an orange-red autofluorescence, and stain intensely with diaminobenzidine (DAB), a marker of endogenous peroxidase activity (Diepen et al., 1954; Creswell et al., 1964; Srebro, 1971; Goldgefter, 1976; Schipper et al., 1988). Histochemical studies have implicated porphyrins and metalloporphyrins (heme) as the source of the autofluorescence and nonenzymatic peroxidase activity in these cells, respectively (Srebro and Cichocki, 1971; Keefer and Christ, 1976; Goldgefter et al., 1980; Kumamoto, 1981; Schipper et al., 1990a).

The role(s) of Gomori astrocytes under normal and pathologic conditions is unknown. In rodent hypothalamus, the astrocytic inclusions accumulate with aging (Schipper et al., 1981) and chronic estrogen exposure (Brawer et al., 1980, 1983). In the hypothalamic arcuate nucleus, chronic estrogenization induces dendritic damage and synaptic remodeling in close proximily

Received Nov. 30, 1990; revised Feb. 22, 1991; accepted Feb. 27, 1991

This work was supported by grants to H.M.S. from the Parkinson Foundation of Canada, the Parkinson's Disease Foundation (U.S.), and the Medical Research Council of Canada. We wish to thank Adina Mateescu-Cantuniari for excellent technical assistance and Rhona Rosenzweig and Sandy Fraiberg for skillful secretarial help.

Correspondence should be addressed to H. M. Schipper, M.D., Ph.D., Lady Davis Institute for Medical Research of the Sir Mortimer B. Davis-Jewish General Hospital, 3755 Cote Ste-Catherine Road, Montreal, Quebec, Canada H3T 1E2.

Copyright (C) 1991 Society for Neuroscience $0270-6474 / 91 / 112170-07 \$ 03.00 / 0$ to heavily granulated Gomori astrocytes (Brawer et al., 1978; Naftolin et al., 1988; Olmos et al., 1989). Because estradiol is metabolized, in part, to 2-hydroxyestradiol (catecholestrogen) in mammalian hypothalamus (Ball and Knuppen, 1978; Maclusky et al., 1981), we hypothesized that glial peroxidase activity oxidizes catecholestrogens to potentially neurotoxic semiquinone radicals analogous to the metabolic fate of estradiol in reproductive tissues (Schipper et al., 1990a). In dissociated rat brain cell cultures, the sulfhydryl agent cysteamine induces the Gomori phenotype in a large proportion of astrocytes, as evidenced by their distinct autofluorescence, endogenous peroxidase activity, and affinity for chrome alum hematoxylin (Schipper et al., 1990b). In the present electron spin resonance (ESR) study, we set out to determine whether the astrocytic peroxidase activity induced by cysteamine promotes the oxidation of the catechols 2-hydroxyestradiol and dopamine to their ortho-(o-) semiquinone derivatives. Because astrocytes in cysteamineexposed primary cultures are histochemically identical to Gomori-positive astrocytes in vivo, positive results in this tier of experiments would implicate the Gomori-positive glia in the pathogenesis of estradiol-related dendritic degeneration and freeradical-related neurologic disorders.

\section{Materials and Methods}

Tissue culture. Primary glial cultures were prepared from 2-d-old SpragueDawley rat brains by mechanoenzymatic dissociation according to the technique of Robbins et al. (1982). Cells were grown in Ham's F-12 and high-glucose Dulbecco's modified Eagle's medium (DMEM) (50: $50 \mathrm{v} / \mathrm{v})$ supplemented with $10 \mathrm{~mm}$ HEPES, $5 \%$ heat-inactivated horse/ $5 \%$ fetal calf sera, and penicillin $(50 \mathrm{U} / \mathrm{ml}) /$ streptomycin $(50 \mu \mathrm{g} / \mathrm{ml})$. Cells $\left(2.8 \times 10^{3}\right.$ per cc medium) were plated on eight-chambered Lab Tek slides and on $35-\mathrm{mm}$ plastic dishes precoated with poly-D-lysine and $50 \% \mathrm{Nu}$ serum. Cultures were incubated at $37^{\circ} \mathrm{C}$ in humidified $95 \%$ air $/ 5 \% \mathrm{CO}_{2}$, and medium was changed twice weekly.

Drug treatments. From 6 to $18 \mathrm{~d}$ in vitro (DIV), cultures received 880 $\mu \mathrm{M}$ of freshly prepared cysteamine (Sigma Chemical Co., St. Louis, MO) with each change of medium or were left untreated. This cysteamine regimen induces the accumulation of autofluorescent, peroxidase-positive inclusions in cultured astroglia identical to those that characterize Gomori-positive astrocytes in the periventricular brain (Schipper et al., $1990 \mathrm{~b})$. Cultures were prepared for histochemical analysis and ESR at 18 DIV.

Autofluorescence, peroxidase histochemistry, and anti-GFAP immunolabeling. For tissue autofluorescence, cysteamine-treated and untreated cultures were examined live and after fixation with $10 \%$ buffered formalin using a Nikon Labophot fluorescent microscope equipped with a $\mathrm{G}$ (green light) excitation filter with main wavelength at $546 \mathrm{~nm}$ (range, $535-550 \mathrm{~nm}$ ) and a spectral transmission range of 550-700+ $\mathrm{nm}$. For endogenous peroxidase activity, additional cultures were fixed in $4 \%$ paraformaldehyde for $30 \mathrm{~min}$ at ronm temperature followed by incubation in modified Karnovsky medium. The latter consists of $50 \mathrm{mg}$ 


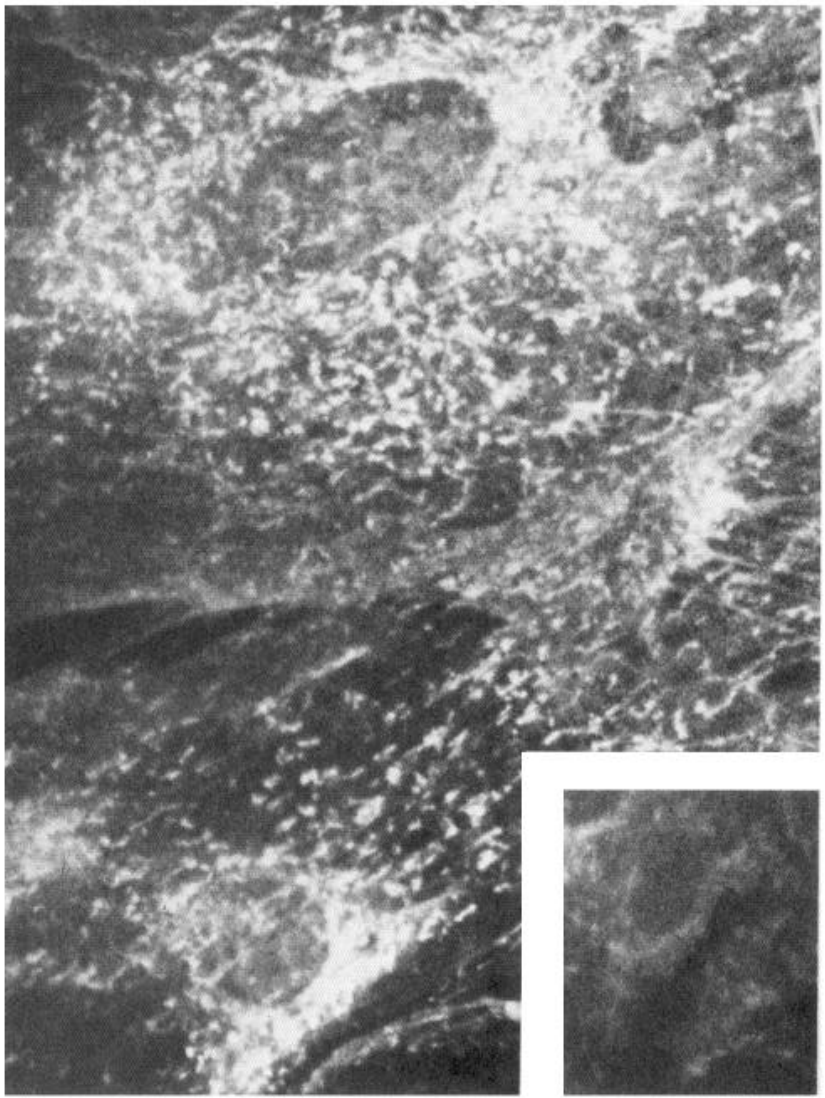

Figure 1. Neonatal day 2 rat neuroglial cultures examined on DIV 18 for tissue autofluorescence: cysteamine-exposed culture exhibiting intense cytoplasmic orange-red autofluorescence consistent with the presence of porphyrin. Inset, Untreated, control culture exhibiting faint or no orange-red autofluorescence. Magnification, $611 \times$.

3,3'-diaminobenzidine (DAB; Sigma) in $100 \mathrm{ml}$ of 0.1 M PBS to which $3 \mathrm{ml}$ of fresh $3 \% \mathrm{H}_{2} \mathrm{O}_{2}$ was added (final $\mathrm{pH}, 6.5$ ). After $30 \mathrm{~min}$ of incubation, the reaction was terminated with PBS, and the cultures were prepared for light microscopy as previously described (Schipper et al., 1990b). To confirm the localization of DAB-positive inclusions to astrocytes, cysteamine-treated cultures were subjected to indirect immunofluorescence using a thoroughly characterized monoclonal antibody (diluted 1:20; provided by Dr. Eugenia Wang) directed against the astrocyte marker glial fibrillary acidic protein (GFAP) following the $\mathrm{DAB}$ procedure. A fluorescein-conjugated goat anti-mouse antibody was used to visualize the GFAP. For controls, GFAP-positive astrocytes devoid of cytoplasmic granules were observed in the absence of DAB. Brown, DAB-positive granules were readily identified, but astrocytes remained unlabeled, following substitution of control ascites fluid for the anti-GFAP antibody.

Electron spin resonance. Electron spin resonance with magnesium spin stabilization was employed to determine whether the cysteamineinduced astrocytic peroxidase activity promotes the oxidation of 2hydroxyestradiol (catecholestrogen) and dopamine to their highly reactive and potentially neurotoxic $O$-semiquinone derivatives.

ESR was performed using a Bruker ER200D-SRC spectrometer operated at X-band and $100-\mathrm{kHz}$ field modulation $(9.75-\mathrm{GHz}$ microwave; 3465-gauss field midrange). Complexation of catechol-derived semiquinones with the diamagnetic cation magnesium stabilizes the former in neutral solutions, thereby facilitating the recording of their ESR spectra (Kalyanaraman et al., 1984, 1985, 1986). For all experiments, spectra obtained at $3 \mathrm{~min}$ incubation time were analyzed.

Spectrometer settings were selected to optimize resolution (see Fig. 4) or to achieve maximum sensitivity when low concentrations of catechol substrate were analyzed (see Fig. 5). Consistent ESR results were obtained from analyses repeated at least four times for each test solution using equivalent amounts of cysteamine-exposed and control culture material.

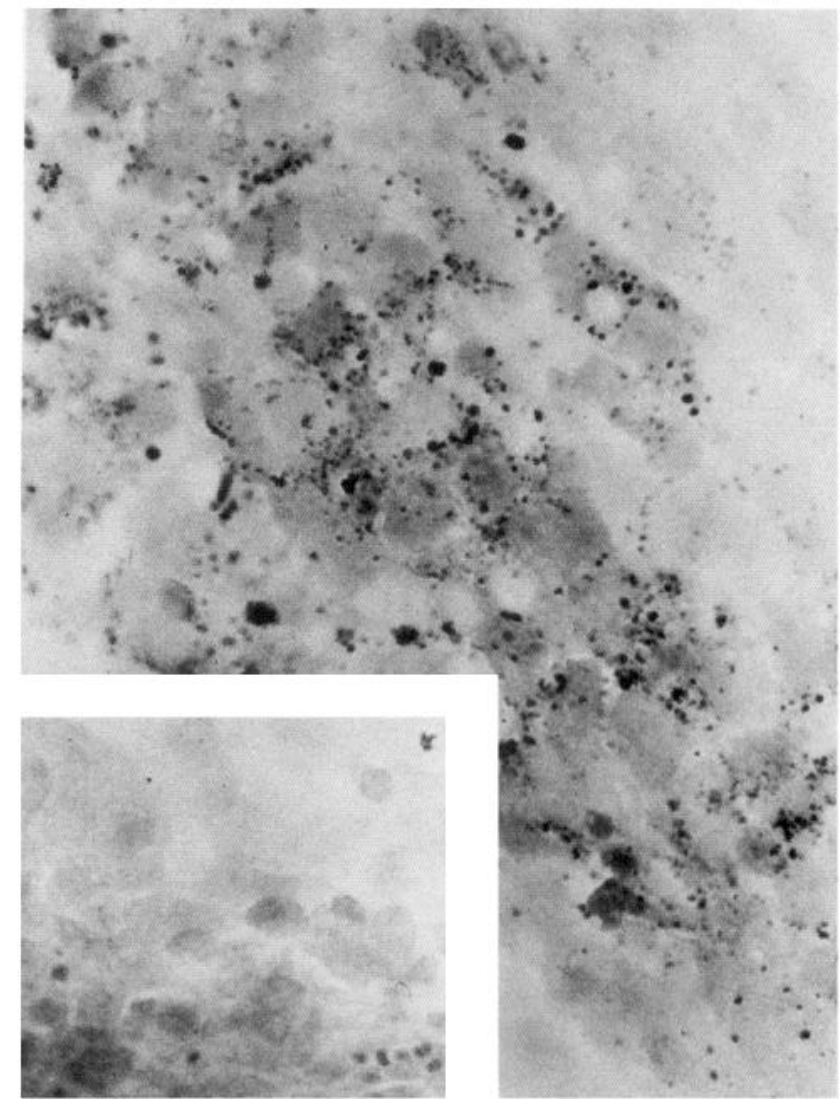

Figure 2. Neonatal day 2 rat glial cultures stained on DIV 18 with $\mathrm{DAB}$ for endogenous peroxidase activity: cysteamine-treated culture exhibiting a massive accumulation of DAB-positive cytoplasmic granules. Methyl green counterstain. Inset, Control culture exhibiting no endogenous peroxidase staining. Magnification, $388 \times$.

At the time of sampling, culture supernatants from individual Lab Tek chambers were replaced with test solutions $(0.4-\mathrm{ml}$ aliquots) containing various concentrations of 2-hydroxyestradiol or dopamine (Sigma) with or without cofactors (NADPH, $\mathrm{H}_{2} \mathrm{O}_{2}$ ) known to drive peroxidase-catalyzed catechol oxidation in cell-free systems (Kalyanaraman et al., 1984, 1985, 1986). 2-Hydroxyestradiol was determined to be $>98 \%$ pure by reverse-phase HPLC. Test solutions were prepared fresh from $10^{-2} \mathrm{M}$ stock solutions containing ethanol solvent diluted more than $10^{5}$-fold. Cell monolayers were disrupted and mixed with test solutions by $15 \mathrm{sec}$ of repetitive pipetting at room temperature, and samples were immediately transferred to quartz flat cells for ESR analysis.

\section{Results}

Autofluorescence, peroxidase histochemistry, and anti-GFAP immunolabeling. Untreated 18-d-old glial cultures exhibited faint or no autofluorescence (Fig. 1, inset) and minimal endogenous peroxidase activity (Fig. 2, inset). In contrast, cysteamine exposure induced a massive accumulation of cytoplasmic inclusions that emit orange-red autofluorescence (Fig. 1) and stain intensely with DAB (Fig. 2). The autofluorescence bleaches rapidly when cultures are examined live but is preserved by formalin fixation analogous to the observations of Srebro and Macinska (1972) in diencephalic explants. We have previously demonstrated that the induced peroxidase activity is nonenzymatic in nature (Schipper et al., 1990b). Using a combined DAB histochemistry-GFAP immunofluorescence technique, the peroxidase-positive granules were noted to localize exclusively to astrocytes (Fig. 3), confirming our previous observations us- 
A

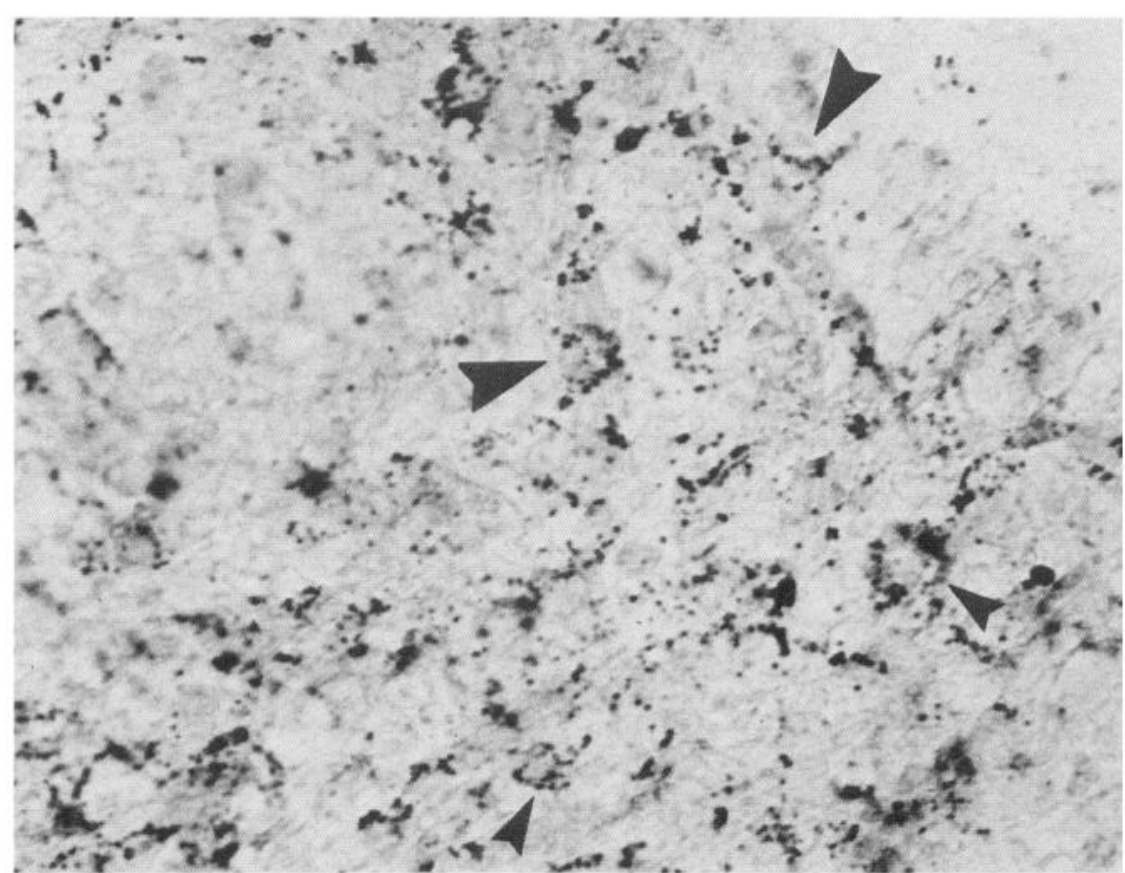

B

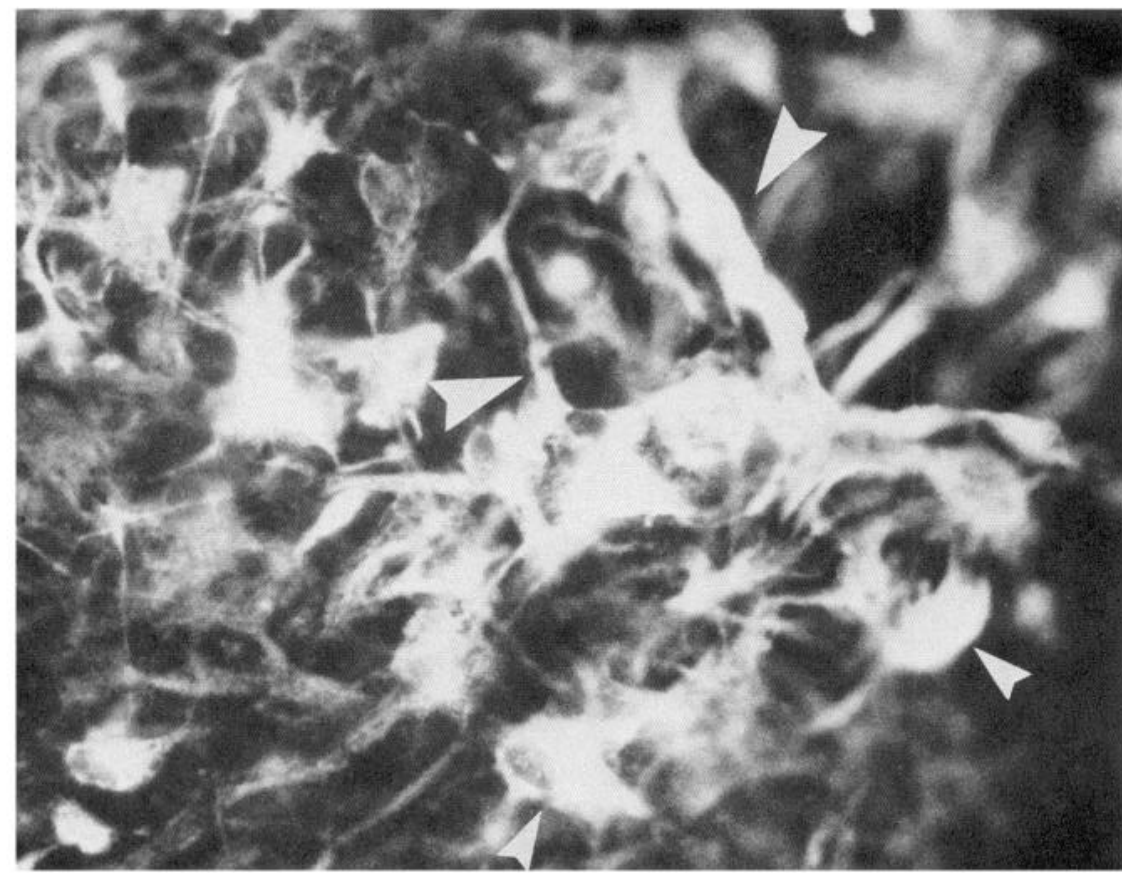

Figure 3. DAB histochemistry-GFAP immunofluorescence of cysteaminetreated glial culture (DIV 18). Peroxidase-positive granules (arrowheads in $A$ ) localize exclusively to GFAP-positive astrocytes $(B$, arrowheads). Magnification, $400 \times$. ing an alternative immunolabeling procedure (Schipper et al., 1990b).

Electron spin resonance. In the catecholestrogen experiment, high-intensity ESR signals specific for the $o$-semiquinone that derives from the autoxidation of 2-hydroxyestradiol $\left(10^{-2} \mathrm{M}\right)$ were observed in cell-free DMEM at alkaline $\mathrm{pH}$ in the presence of $\mathrm{MgCl}_{2}(0.5 \mathrm{M}$; Fig. $4 A)$. The hyperfine coupling constants for the $o$-semiquinone spectra $\left(\mathrm{A}^{\mathrm{H}}{ }_{\mathrm{C} 6}=8.42,5.66\right.$ gauss; $\mathrm{A}^{\mathrm{H}}{ }_{C 9}=9.43$ gauss; $\mathrm{A}_{\text {aromatic }}^{\mathrm{H}}=0.30$ gauss) were in close agreement with those reported by Kalyanaraman et al. (1984, 1985, 1986). Incuba- tions with steroid-free vehicle yielded no free-radical species in any of the experiments. Using identical ESR parameter settings, repeated incubations of equimolar concentrations of 2-hydroxyestradiol with untreated (control) cell homogenates and appropriate cofactors $\left(0.3 \mathrm{M}\right.$ NADPH, $\left.0.1 \mathrm{~mm} \mathrm{H}_{2} \mathrm{O}_{2}\right)$ at neutral $\mathrm{pH}$ yielded $o$-semiquinone spectra of very low amplitude (Fig. $4 B)$. In striking contrast to the meager pro-oxidant capacity of the control cultures, very intense $o$-semiquinone spectra (with amplitudes four to six times greater than those obtained from control cultures) indicative of robust catechol oxidation were 
A
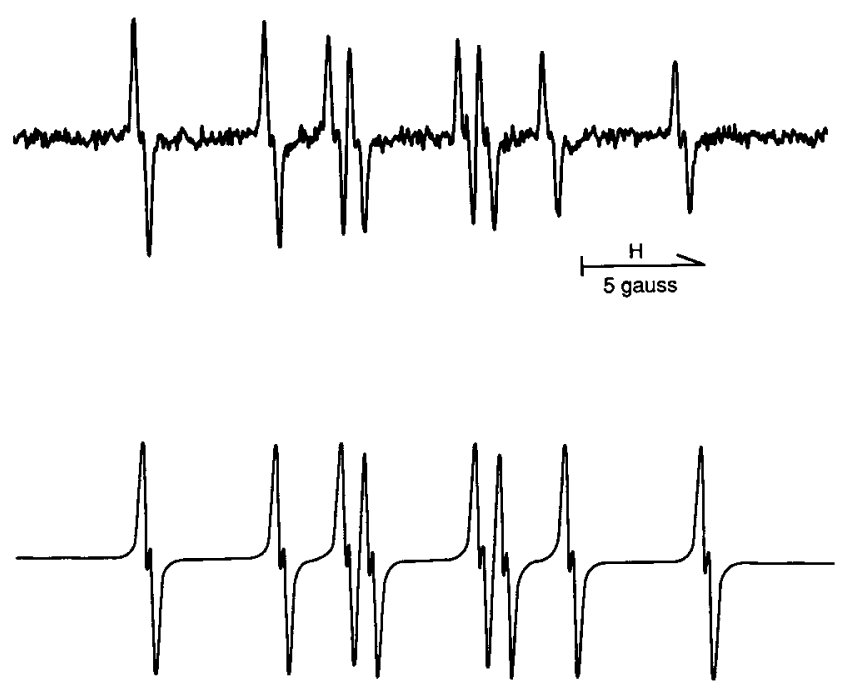

B
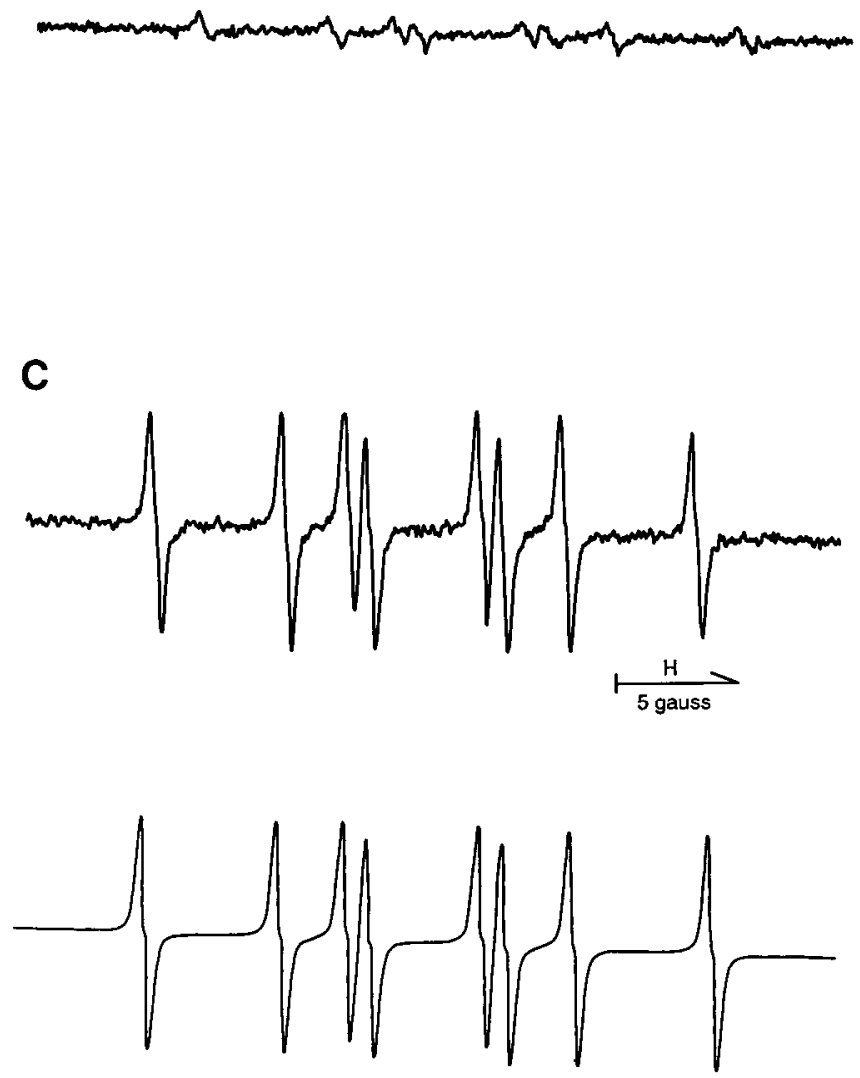

Figure 4. ESR spectra of metal-complexed semiquinones from the peroxidase- $\mathrm{H}_{2} \mathrm{O}_{2}$ oxidation of 2-hydroxy(catechol)estradiol. ESR spectra were obtained with a Bruker ER200D SRC spectrometer operated in the X-band. TE012 mode resonant cavity was used with a flat sample cell made of quartz. Instrumental settings: $100-\mathrm{kHz}$ field-modulation consistently generated at neutral $\mathrm{pH}$ following incubation of equimolar concentrations of 2-hydroxyestradiol with homogenates obtained from cysteamine-pretreated (peroxidase-enriched) astrocyte monolayers (Fig. $4 \mathrm{C}$ ). A marked reduction in signal amplitude was observed in the absence of $\mathrm{H}_{2} \mathrm{O}_{2}$ substrate (Fig. 5). Finally, NADPH boosted, but was not an absolute requirement for, glial peroxidase-catalyzed catechol oxidation (Fig. 5D).

As in the case of 2-hydroxyestradiol, magnesium-stabilized $O$-semiquinone spectra resulting from the autoxidation of dopamine (1 mM) were readily detected in cell-free DMEM at $\mathrm{pH}$ 10.0 (Fig. $6 A$ ). The hyperfine coupling constants $\left(\mathrm{A}^{\mathrm{H}} \mathrm{CH}^{2}=3.35\right.$ gauss; $\mathrm{A}_{\text {aromatic }}=4.03,0.72$ gauss) for the dopamine-o-semiquinone spectra are similar to those reported in the literature (Pedersen, 1985). Incubation of dopamine with cysteamine-pretreated (peroxidase-enriched) astrocyte cultures and appropriate cofactors at neutral $\mathrm{pH}$ consistently produced ESR spectra with amplitudes two to three times greater than those obtained from the untreated monolayers under otherwise identical conditions (Fig. 6B, C). As in the catecholestrogen study, exogenous NADPH augmented, but was not an absolute prerequisite for, the dopamine oxidation reaction (data not shown).

\section{Discussion}

Treatment of dissociated rat brain cell cultures with cysteamine resulted in the accumulation of autofluorescent astrocytic cytoplasmic granules that stained intensely with the peroxidase marker DAB. These histochemical properties are identical to those that characterize Gomori-positive astrocytes residing in subependymal regions and circumventricular organs of the vertebrate nervous system (Srebro, 1971; Goldgefter et al., 1980; Kumamoto, 1981; Schipper et al., 1990a). The analysis of the fluorescence spectra and histochemical profile of the peroxidase activity suggests that cysteamine induces the accumulation of metalloporphyrins (probably heme) in these cells (Schipper $1990 \mathrm{a}, \mathrm{b}$ ). By virtue of redox changes in their metal cores, metalloporphyrins mediate nonenzymatic peroxidase reactions

width, 0.25 gauss; incident microwave power, $2 \mathrm{~mW}$; microwave frequency, $9.75 \mathrm{GHz}$. The external magnetic field was swept over a range of 30 or 40 gauss, and the sweep for each scan was 50 sec with a 0.05 sec time constant. Sample solutions were prepared at room temperature and introduced to the sample cell immediately after mixing. $A$ : Top, Autoxidation of 2-hydroxyestradiol in serum-free medium in the absence of cells following alkalinization to $\mathrm{pH} 10.0$ with $\mathrm{NaOH}$. Medium contained 2-hydroxyestradiol $\left(10^{-2} \mathrm{M}\right), \mathrm{MgCl}_{2}(0.5 \mathrm{M})$, and $\mathrm{NaOH}$ in DMEM. The characteristic $o$-semiquinone spectrum is identical to that observed by Kalyanaraman et al. (1984). Bottom, Computer-simulated spectrum of the 2-hydroxyestradiol $O$-semiquinone derived from the measured hyperfine coupling constants given in the text. $B$, Incubation of 2-hydroxyestradiol $\left(10^{-2} \mathrm{M}\right), \mathrm{MgCl}_{2}(0.5 \mathrm{M})$, NADPH $(0.3 \mathrm{M})$, and $\mathrm{H}_{2} \mathrm{O}_{2}(0.1 \mathrm{~mm})$ with tissue homogenate derived from untreated (control) brain cell culture (pH, 7.0). The gain settings in $B$ and $C$ are identical, permitting direct amplitude comparisons. $C: T o p$, Incubation as in $B$ with tissue homogenate derived from cysteamine-pretreated (peroxidase-enriched) brain cell culture. An intense $o$-semiquinone signal is observed with hyperfine structure identical to the pattern obtained in the cell-free 2-hydroxyestradiol autoxidation experiment $(A)$. (The slight broadening of the peaks from 0.25 gauss in $A$ to 0.32 gauss in $C$ is due to the increased viscosity of test solutions containing cell homogenates.) The peroxidase activity induced in astrocytes by cysteamine catalyzes catechol oxidation to $o$-semiquinone radicals. Bottom, Computer-simulated spectrum of the 2-hydroxyestradiol $o$-semiquinone using coupling constants derived from $C$, top. 
A
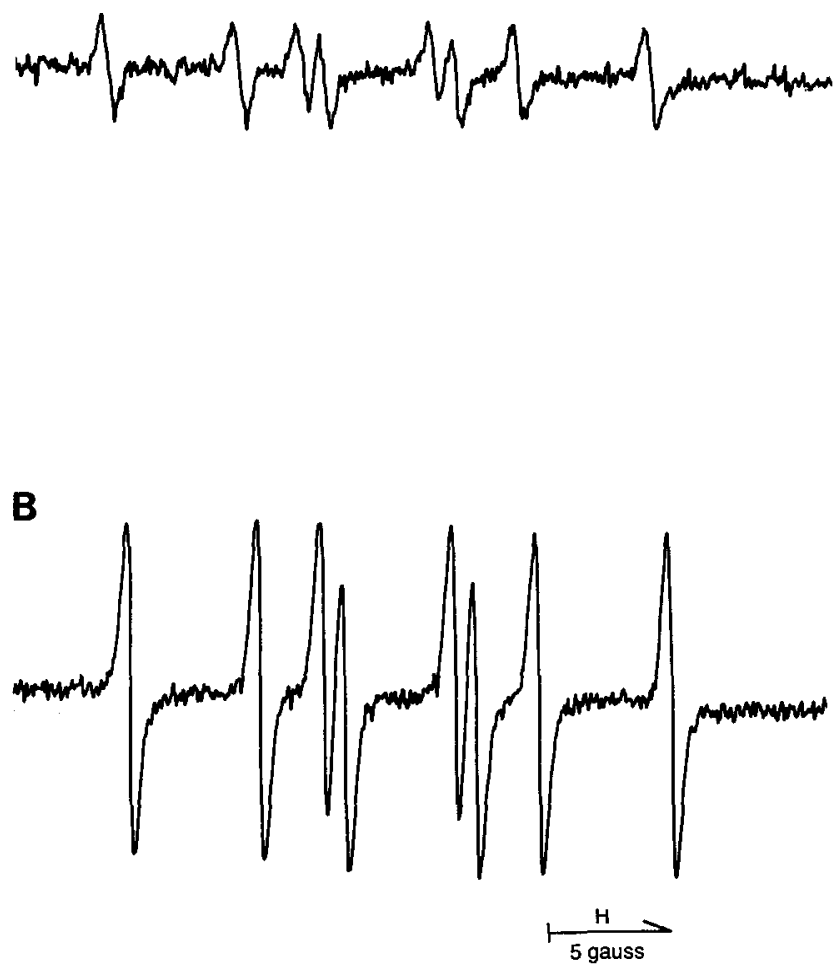

C

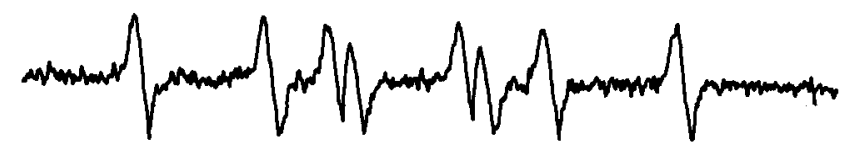

D

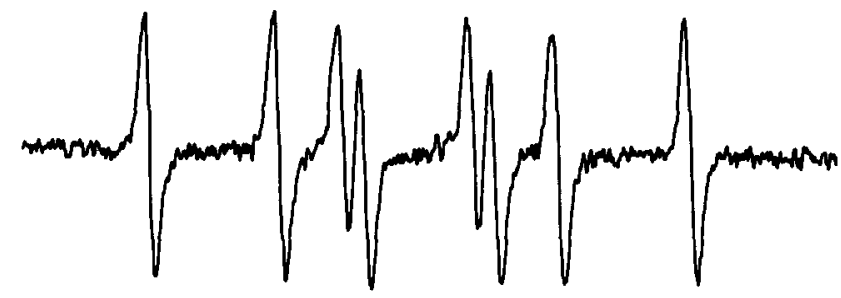

Figure 5. Effects of $\mathrm{H}_{2} \mathrm{O}_{2}$ substrate and NADPH on astrocyte peroxidase-catalyzed oxidation of 2-hydroxyestradiol. $A$, Autoxidation of 2-hydroxyestradiol $\left(10^{-4} \mathrm{M}\right)$ in serum-free medium $(\mathrm{pH}, 10.0)$, under conditions identical to those described in Figure 4. B, Incubation of 2-hydroxyestradiol $\left(10^{-4} \mathrm{M}\right), \mathrm{MgCl}_{2}(0.5 \mathrm{M}), \mathrm{NADPH}(0.3 \mathrm{M})$, and $\mathrm{H}_{2} \mathrm{O}_{2}$ $(0.1 \mathrm{~mm})$ with cysteamine-treated astrocyte culture at $\mathrm{pH} 7.0$. The gain settings in $B-D$ are identical, permitting direct amplitude comparisons. identical to those observed in Gomori astrocyte granules (Askarov et al., 1984; Van Steveninck et al., 1987). The results of the present study indicate that cysteamine-induced astrocyte peroxidase activity promotes the activation of catechols to semiquinone radicals. Using ESR with $\mathrm{Mg}^{2+}$ spin stabilization, highintensity signals specific for the $o$-semiquinone derived from the spontaneous autoxidation of 2-hydroxyestradiol (Kalyanaraman et al., 1984, 1985, 1986) were observed at alkaline $\mathrm{pH}$ in the absence of cells. Incubation of equimolar concentrations of 2-hydroxyestradiol with untreated (control) cell homogenates and appropriate cofactors at neutral $\mathrm{pH}$ yielded barely detectable $o$-semiquinone spectra. The pseudoperoxidase activity accruing to low levels of glial metalloporphyrins normally present in untreated 18-d-old brain cell cultures (Schipper et al., 1990b), some degree of autoxidation that occurs at neutral $\mathrm{pH}$, and the enzymatic activities of various oxidases and peroxidases (Graham, 1978; Graham et al., 1978; Rosenberg, 1988) may all contribute to the low level of catechol oxidation in the control cell incubations. In contrast to the control cultures, very intense $o$-semiquinone spectra indicative of robust catechol oxidation were consistently generated at neutral $\mathrm{pH}$ following incubation of equimolar concentrations of 2-hydroxyestradiol with homogenates obtained from cysteamine-pretreated (peroxidaseenriched) astrocyte monolayers. The marked reduction in signal amplitude observed in the absence of $\mathrm{H}_{2} \mathrm{O}_{2}$ substrate further attests to the important role of the glial peroxidase activity in the augmentation of catecholestrogen oxidation in this system. In cell-free systems, exogenous NADPH is an important cofactor for peroxidase-catalyzed oxidations (Kalyanaraman et al., $1984,1985,1986)$. In the present study, NADPH enhanced, but was not an absolute prerequisite for, glial peroxidase-catalyzed catechol oxidation. Presumably, the high sulfhydryl content of Gomori-positive granules (Srebro, 1971; Goldgefter, 1976) or equivalent reducing substances sustain peroxidase-mediated reactions in these brain cell preparations.

In adult rodents, chronic estrogenization markedly increases numbers of peroxidase (Gomori)-positive granules in astrocytes of the hypothalamic arcuate nucleus (Brawer et al., 1978, 1980, 1983; Schipper et al., 1990a). In these estrogenized animals, degenerating dendrites and pathologic myelin figures are frequently seen in close apposition to astrocytic processes replete with Gomori-positive inclusions (Brawer et al., 1978). The results of the present ESR experiments indicate that the nonenzymatic peroxidase activity endogenous to Gomori astrocytes in capable of promoting the oxidation of catecholestrogens to potentially neurotoxic free-radical intermediates. These data are consistent with a previous report that subcellular fractions of rabbit hypothalamus contain hemoproteins with peroxidatic activity capable of catalyzing 2- and 4-hydroxylation of estradiol (Mondschein et al., 1986). While the high thiol content of the Gomori astrocyte inclusions may confer some degree of cytoprotection by serving as a "sink" for estrogen-derived free-radical species (Jellinck and Fletcher, 1970; Maggs et al., 1983; Abdul-Hajj and Cisek, 1986, 1988), leakage of free radicals into

$C$, Incubation as in $B$ in the absence of $\mathrm{H}_{2} \mathrm{O}_{2}$ substrate. A marked reduction in signal amplitude is observed relative to $B$. $D$. Incubation as in $B$ in the absence of NADPH. NADPH augments, but is not an absolute requirement for, catechol oxidation in this system. ESR measurement parameters are as in Figure 4. 
A
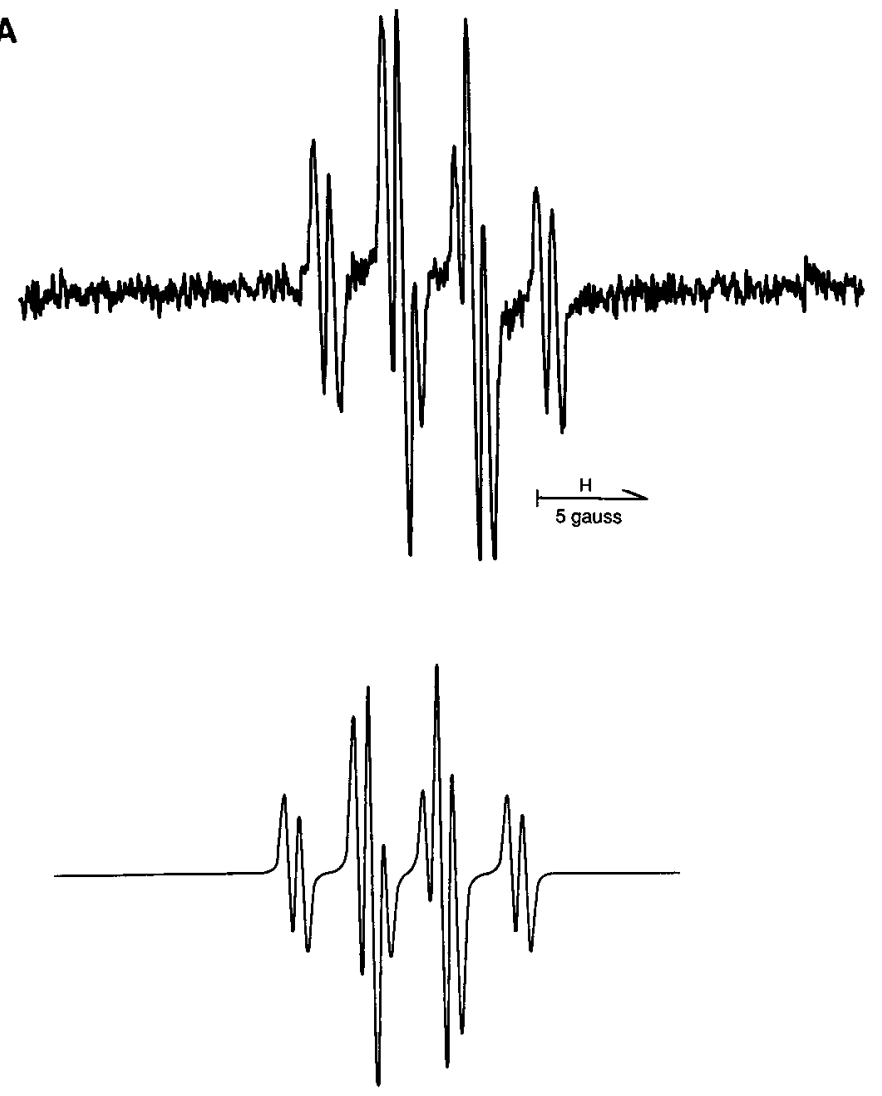

B
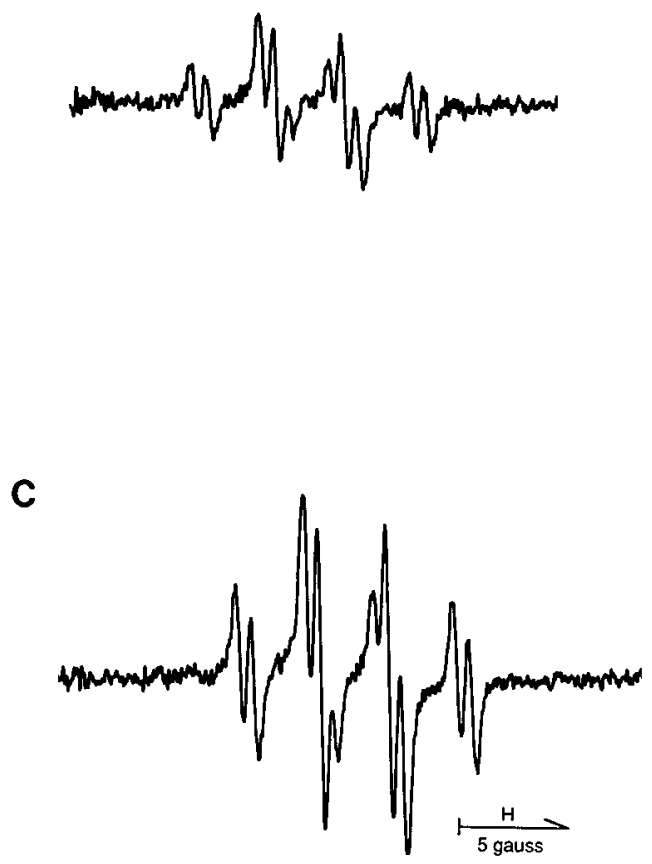

Figure 6. ESR spectra of magnesium complexed semiquinones derived from the peroxidase $-\mathrm{H}_{2} \mathrm{O}_{2}$ oxidation of dopamine. $A$ : Top, Autoxidation of dopamine in serum-free medium in the absence of cells at $\mathrm{pH} 10.0$. Medium contained dopamine $(1 \mathrm{~mm}), \mathrm{MgCl}_{2}(0.2 \mathrm{M})$, and $\mathrm{NaOH}$ in the adjacent neuropil may induce lipid peroxidation and the degeneration of nearby dendrites and other vulnerable neuronal constituents (Kontos et al., 1985).

As in the case of 2-hydroxyestradiol, incubation of dopamine with cysteamine-pretreated (peroxidase-enriched) astrocyte cultures and appropriate cofactors produced ESR spectra with consistently greater amplitudes than those obtained from the untreated astrocyte monolayers. These findings suggest that the nonenzymatic peroxidase activity of Gomori-positive astrocytes in vivo may play an important role in CNS aging and in the pathogenesis of free-radical-related neurologic disorders. In idiopathic and neurotoxin-induced parkinsonism, accelerated turnover of dopamine in surviving nigrostriatal neurons may generate excessive amounts of toxic semiquinones (dopaquinones) and oxyradicals. The latter, in turn, may perpetuate cascades of nigral cell damage and progressive clinical deterioration (Barbeau, 1984; Cohen, 1987). Although the role of glial monoamine oxidases in catecholamine oxidation is well established (Weiner and Molinoff, 1989), ESR studies indicate that norepinephrine and dopamine derivatives in vitro are readily oxidized to semiquinones with proven neurotoxic activity via peroxidase-mediated reactions (Metodiewa et al., 1989). In light of the present findings, it is conceivable that the peroxidase activity of glial metalloporphyrins may exacerbate parkinsonism and other free-radical-related neurologic disorders by augmenting the bioactivation of endogenous catechols and environmentally derived xenobiotics to neurotoxic intermediates. Furthermore, the age-dependent increases in numbers of Gomori-positive astrocytes that have been documented in rodent and human brain (Schipper et al., 1981, 1988) may explain, at least in part, the enhanced vulnerability of the senescent nervous system to idiopathic and neurotoxin-induced parkinsonism (Barbeau, 1984) and perhaps other free-radical-related neurodegenerations.

\section{References}

Abdul-Hajj Y, Cisek P (1986) Regioselective reaction of thiols with catechol estrogens and estrogen-o-semiquinones. J Steroid Biochem 25:245-247.

Abdul-Hajj Y, Cisek P (1988) Catechol estrogen adducts. J Steroid Biochem 31:107-110.

Askarov KA, Tsoi GG, Onishchenko VA, Simonova LY, Kavoz-Ogly AA, Rish MA (1984) Catalase and peroxidase activity of porphyrins. Biokhimiia 49:754-757.

Ball P, Knuppen R (1978) Formation of 2- and 4-hydroestrogens by brain, pituitary, and liver of the human fetus. J Clin Endocrinol Metab 47:732-737.

Barbeau A (1984) Etiology of Parkinson's disease: a research strategy. Can J Neurol Sci 11:24-28.

Brawer J, Naftolin F, Martin J, Sonnenschein C (1978) Effects of a single injection of estradiol valerate on the hypothalamic arcuate nucleus and on reproductive function in the female rat. Endocrinology 103:501-512.

DMEM. The characteristic dopamine-o-semiquinone spectrum is observed (Pedersen, 1985). Bottom, Computer-simulated spectrum of the dopamine- $O$-semiquinone derived from the measured hyperfine coupling constants given in the text. $B$. Incubation of dopamine (1 $\mathrm{mm}$ ), $\mathrm{MgCl}_{2}(0.2 \mathrm{M})$, NADPH $(0.3 \mathrm{M})$, and $\mathrm{H}_{2} \mathrm{O}_{2}(0.1 \mathrm{~mm})$ with tissue homogenate derived from an untreated (control) astrocyte culture $(\mathrm{pH}$, 7.0). The gain settings in $B$ and $C$ are identical. $C$, Incubation as in $B$ with tissue derived from cysteamine-pretreated (peroxidase-enriched) astrocyte culture. ESR spectra amplitudes are approximately 2.5 -fold greater than those observed in $B$. The cysteamine-induced peroxidase activity catalyzes catechol oxidation to $o$-semiquinone radicals. ESR measurement parameters are as in Figure 4. 
Brawer J, Schipper H, Naftolin F (1980) Ovary-dependent degeneration in the hypothalamic arcuate nucleus. Endocrinology 107:274 279.

Brawer J, Schipper H, Robaire B (1983) Effects of long term androgen and estradiol exposure on the hypothalamus. Endocrinology 112:194199

Cohen G (1987) Oxygen radicals and Parkinson's disease. Upjohn Symposium on Oxygen Radicals (Halliwell B, ed.), pp 130-135. Augusta, MI.

Creswell GF, Reis DJ, Maclean P (1964) Aldehyde fuchsin-positive material in brain of squirrel monkey (Saimiri sciureus). Am J Anat 115:543-558.

Diepen R, Engelhardt F, Smith-Agreda V (1954) Über Ort und Art der Entstehung des Neurosekrets im supraoptico-hypophysären System bei Hund und Katze. Verh Anat Ges Anat Anz [Suppl] 101:276286.

Goldgefter L (1976) Studies on the structure and function of Gomoripositive glial cells in the rat hypothalamus. Acta Anat (Basel) 95:545557.

Goldgefter L, Schejter AS, Gill D (1980) Structural and microspectrofluorometric studies on glial cells from the periventricular and arcuate nuclei of the rat hypothalamus. Cell Tissue Res 211:503-510.

Graham D, Tiffany S, Bell W Jr, Gutknecht W (1978) Autoxidation versus covalent binding of quinones as the mechanism of toxicity of dopamine, 6-hydroxydopamine, and related compounds towards C1300 neuroblastoma cells in vitro. Mol Pharmacol 14:644-653.

Graham DG (1978) Oxidative pathways for catecholamines in the genesis of neuromelanin and cytotoxic quinones. Mol Pharmacol 14: 633-643.

Jellinck PH, Fletcher R (1970) Peroxidase-catalyzed conjugation of $\left[4-{ }^{14} \mathrm{C}\right]$-estradiol with albumin and thiols. Can J Biochem 48:11921198 .

Kalyanaraman B, Sealy R, Sivarajah K (1984) An electron spin resonance study of $o$-semiquinous formed during the enzymatic and autoxidation of catechol estrogens. J Biol Chem 259:14018-14022.

Kalyanaraman B, Felix CC, Sealy RC (1985) Semiquinone anion radicals of catechol(amine)s, catechol estrogens, and their metal ion complexes. Environ Health Perspect 64:185-198.

Kalyanaraman B, Hintz P, Sealy R (1986) An electron spin resonance study of free radicals from catechol estrogens. Fed Proc 45:24772484.

Keefer DA, Christ JF (1976) Distribution of endogenous diaminobenzidine-staining cells in the normal rat brain. Brain Res 116:312316.

Kontos H, Wei W, Ellis E, Jenkins L, Povlishock J, Rowe G, Hess M (1985) Appearance of superoxide anion radical in cerebral extracellular space during increased prostaglandin synthesis in cats. Circ Res 57:142-151.

Kumamoto $T$ (1981) Histochemical study on endogenous diaminobenzidine-positive granules in the glia cell of rat brain. Acta Histochem Cytochem 14:173-185.
Maclusky N, Naftolin F, Krey L, Franks S (1981) The catecholestrogens. J Steroid Biochem 15:111-124.

Maggs JL, Grabowski PS, Park BK (1983) Drug protein conjugatesIII. Inhibition of the irreversible binding of ethinylestradiol to rat liver microsomal protein by mixed function oxidase inhibitor ascorbic acid and thiols. J Steroid Biochem 19:1273-1278.

Metodiewa D, Reszka K, Dunford H (1989) Evidence for a peroxidatic oxidation of norepinephrine, a catecholamine, by lactoperoxidase. Biochem Biophys Res Commun 160:1183-1188.

Mondschein J, Hersey R, Weisz J (1986) Puritication and characterization of estrogen-2/4-hydroxylase activity from rabbit hypothalamus: peroxidase-mediated catechol estrogen formation. Endocrinology 119:1105-1112.

Naftolin F, Maclusky N, Leranth C, Sakamoto H, Garcia-Segura L (1988) The cellular effects of estrogens on neuroendocrine tissues. $J$ Steroid Biochem 30:1-6.

Olmos G, Naftolin F, Perez J, Tranque P, Garcia-Segura L (1989) Synaptic remodeling in the rat arcuate nucleus during the cstrous cycle. Neuroscience 32:663-667.

Pedersen J (1985) EPR spectra from natural and synthetic quinones and quinols. Boca Raton, FL: CRC

Robbins RJ, Sutton RE, Reichlin S (1982) Effects of neurotransmitters and cyclic AMP on somatostatin release from cultured cerebral cortical cells. Brain Res 234:377.

Rosenberg P (1988) Catecholamine toxicity in cerebral cortex in dissociated cell culture. J Neurosci 8:2887-2894.

Schipper H, Brawer JR, Nelson JF, Felicio LS, Finch CE (1981) Role of the gonads in the histologic aging of the hypothalamic arcuate nucleus. Biol Reprod 25:413-419.

Schipper HM, Stopa EG, Wheelock T (1988) Gomori-positive periventricular glia in the human brain. Soc Neurosci Abstr 14:584.

Schipper HM, Lechan RM, Reichlin S (1990a) Glial peroxidase activity in the hypothalamic arcuate nucleus: effects of estradiol valerate-induced persistent estrus. Brain Res 507:200--207.

Schipper HM, Scarborough D, Lechan R, Reichlin S (1990b) Gomoripositive astrocytes in primary culture: effects of in vitro age and cysteamine exposure. Dev Brain Res 54:71-79.

Srebro Z (1971) Periventricular Gomori-positive glia in brains of $x$ irradiated rats. Brain Res 35:463-468.

Srebro Z, Cichocki T (1971) A system of periventricular glia in brain characterized by large peroxisome-like organelles. Acta Histochem Bd 41:108-114.

Srebro Z, Macinska A (1972) Cytochemical demonstration of ferric iron and fluorescence microscopy observations on Gomori-positive glia grown in vitro. Brain Res 42:53-58.

Van Steveninck J, Boegheim JP, Dubbleman TM, Van der Zee J (1987) The mechanism of potentiation of horseradish peroxidase-catalyzed oxidation of NADPH by porphyrins. Biochem J 242:611-613.

Weiner N, Molinoff P (1989) Catecholamines. In: Basic neurochemistry, 4th ed (Siegel G, Agranoff B, Albers R, Holinoff P, eds), pp 233251. New York: Raven. 